\title{
Diffuse Painful Plaques in the Setting of Chronic Lymphocytic Leukemia
}

\author{
Joseph Tadros, MD; Cody Chastain, MD; Eric Tkaczyk, MD, PhD
}

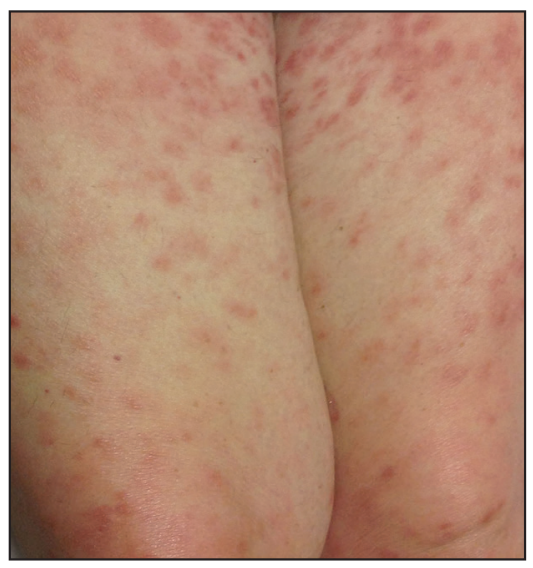

A woman in her seventies presented with a painful rash of 3 months' duration, as well as fever, diaphoresis, and arthralgia of 3 weeks' duration. The rash first appeared on the trunk with centrifugal spread to the face, arms, legs, palms, and soles. The patient had a 16-year history of untreated chronic lymphocytic leukemia, type 2 diabetes mellitus, and hypertension. Physical examination revealed reddish brown, edematous and indurated, coalescing, circular, $1-$ to $1.5-\mathrm{cm}$ plaques on the face (leading to mild leonine facies), upper back, posterior thighs (top), buttocks (bottom), and palms. The arms, legs, abdomen, and chest also were involved to a lesser extent. The skin was diffusely tender to palpation with no accentuation over the lesions. There was no associated pruritus or lymphadenopathy. The patient was febrile (temperature, $38.4^{\circ} \mathrm{C}$ ). A complete blood cell count with differential and liver function tests were within reference range on laboratory evaluation. Blood cultures were negative for both bacterial and fungal infections and finalized after 5 days. Polymerase chain reaction for ehrlichiosis was negative, and histoplasma and cryptococcal antigens were not detected. Computed tomography revealed a hepatic mass, splenomegaly, diffuse adenopathy, and bilateral pulmonary nodules but no parenchymal disease. Skin biopsies were obtained from the left forearm and forehead. Samples were sent to the National Hansen's Disease (Leprosy) Program given concern for Hansen disease based on her husband's history of prior employment in a leprosy hospital.

\section{WHAT'S YOUR DIAGNOSIS?}
a. amyloidosis
b. cutaneous Mycobacterium avium-intracellulare complex infection
c. leukemia cutis
d. Mycobacterium leprae infection
e. palisaded neutrophilic granulomatous dermatitis

PLEASE TURN TO PAGE E19 FOR THE DIAGNOSIS

\footnotetext{
Dr. Tadros is from the University of Cincinnati College of Medicine, Ohio. Drs. Chastain and Tkaczyk are from the Vanderbilt University Medical Center, Nashville, Tennessee. Dr. Chastain is from the Department of Medicine, and Dr. Tkaczyk is from the Department of Dermatology. Dr. Tkaczyk also is from the Department of Veteran Affairs, Tennessee Valley Health System Nashville Campus.

The authors report no conflict of interest.

This work was supported by NIH K12 CA090625 and Career Development Award Number IK2 CX001785 from the US Department of Veterans Affairs Clinical Science R\&D Service.

Correspondence: Eric Tkaczyk, MD, PhD, One Hundred Oaks, 719 Thompson Ln, Ste 26300, Nashville, TN 37204 (eric.tkaczyk@vumc.org). doi:10.12788/cutis.0075
} 


\section{THE DIAGNOSIS:}

\section{Cutaneous Mycobacterium avium-intracellulare Complex Infection}

H istopathologic evaluation revealed superficial and deep perivascular and periadnexal inflammation. The epidermis exhibited some vacuolar interface change and effacement with relatively sparse dyskeratotic cells. A lymphohistiocytic inflammatory infiltrate surrounded the blood vessels, nerves, and adnexal structures and extended into the subcutaneous fat (Figure). Acid-fast, Grocott-Gomori methenamine-silver, Gram, Fite, Treponema pallidum, and Alcian blue stains were performed at our institution and were all negative. Biopsies sent to the National Hansen's Disease (Leprosy) Program demonstrated scattered extracellular acid-fast organisms on Fite staining in the specimen of the forearm. Polymerase chain reaction testing for Mycobacterium leprae DNA was negative. DNA sequencing of the 16S ribosomal RNA gene matched Mycobacterium avium-intracellulare complex (MAC). In the workup of the hepatic mass, the patient incidentally was found to have large-cell transformation of chronic lymphocytic leukemia (CLL) and therefore was treated with bendamustine and rituximab as an outpatient. The patient received 1 chemotherapy infusion every 4 weeks for a total of 10 rounds. At 10-week follow-up after 2 rounds of chemotherapy, all of the skin lesions had resolved despite no antibiotic therapy for atypical infections.

Disseminated infection with MAC is relatively rare in healthy as well as immunocompromised individuals. Clinical disease most commonly is seen as an opportunistic infection in patients with AIDS who have CD4 counts less than $50 / \mathrm{mm}^{3}$ (reference range, $500-1400 / \mathrm{mm}^{3}$ ) or in those with preexisting lung disease. ${ }^{1}$ Cutaneous involvement has been observed in only $14 \%$ of non-AIDS patients with disseminated MAC infection. ${ }^{2}$ In another study of 76 patients with MAC infection, only 2 involved the skin or soft tissue. ${ }^{3}$ Infection of the skin without concurrent pulmonary MAC infection is rare, though trauma may cause isolated skin infection. The cutaneous presentation of MAC infection is highly variable and may include erythematous papules, pustules, panniculitis, infiltrated plaques, verrucous lesions, and draining sinuses. ${ }^{3}$ The lesions have been reported to be painful. ${ }^{1}$

Cutaneous findings occur in up to $25 \%$ of patients with CLL, either due to the seeding of leukemic cells or other secondary lesions. ${ }^{4}$ Leukemia cutis, or skin involvement by B-cell CLL, most commonly presents in the head and neck region as chronic and relapsing erythematous papules and plaques. ${ }^{5}$ It histologically presents as monomorphic lymphocytic infiltrates accentuated around periadnexal and perivascular structures, with some extending into adipose tissue. ${ }^{2}$ In our case, histopathology

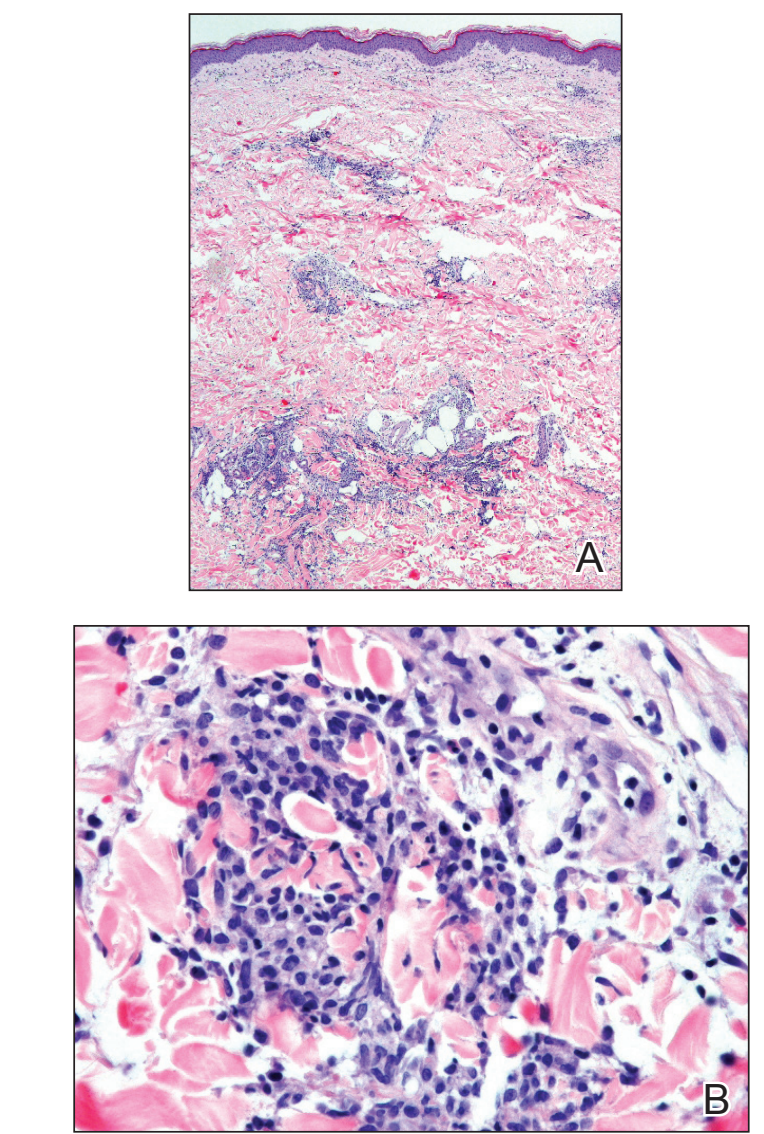

Mycobacterium avium-intracellulare complex infection. A and B, An excisional biopsy specimen from the left forearm showed a lymphohistiocytic inflammatory infiltrate surrounding the blood vessels, nerves, and adnexal structures that extended into the subcutaneous fat $(H \& E$, original magnifications $\times 4$ and $\times 200$ ).

demonstrated a lack of monomorphous infiltrate and thus was inconsistent with leukemia cutis. Similarly, lack of pale pink deposits and lack of neutrophilic infiltrates or degenerated collagen makes amyloidosis and palisaded neutrophilic granulomatous dermatitis incorrect diagnoses, respectively.

We hypothesize that the initially undetected worsening of CLL resulted in an immunocompromised state, which facilitated this unique presentation of cutaneous MAC infection in a human immunodeficiency virusnegative patient with no clinical symptoms of active pulmonary disease. The rash was the presenting sign of both the cutaneous MAC infection and worsening CLL. Additionally, our patient's cutaneous MAC facial 
involvement clinically resembled the leonine facies that is classic in lepromatous leprosy. Rare reports have been published addressing this similarity. ${ }^{6}$

Treatment of MAC pulmonary disease usually includes a combination of clarithromycin or azithromycin, rifampin, and ethambutol (for nodular/bronchiectatic disease), with or without amikacin or streptomycin. ${ }^{7}$ For limited pulmonary disease in patients with adequate pulmonary reserve, surgical resection may be considered in combination with the multidrug MAC pulmonary treatment regimen for 3 months to 1 year. Patients with localized MAC disease involving only the skin, soft tissue, tendons, and joints usually are treated with surgical excision in combination with clarithromycin, rifampin, and ethambutol for 6 to 12 months. ${ }^{7}$ In our patient, we believe that chemotherapy and the subsequent reconstituted immune system likely cleared the MAC infection without targeted antibiotic treatment.

Acknowledgments-The authors would like to thank David Scollard, MD, PhD, and Barbara Stryjewska, MD, from the National Hansen's Disease (Leprosy) Association (Baton Rouge, Louisiana).

\section{REFERENCES}

1. Robak E, Robak T. Skin lesions in chronic lymphocytic leukemia. Leuk Lymphoma. 2007;48:855-865.

2. Plaza JA, Comfere NI, Gibson LE, et al. Unusual cutaneous manifestations of chronic lymphocytic leukemia. J Am Acad Dermatol. 2009;60:772-780

3. Sivanesan SP, Khera P, Buckthal-McCuin J, et al. Cutaneous Mycobacterium avium-intracellulare complex associated with immune reconstitution inflammatory syndrome. J Am Acad Dermatol. 2010;62:E25-E26.

4. Horsburgh CR, Mason UG, Farhi DC, et al. Disseminated infection with Mycobacterium avium-intracellulare. a report of 13 cases and a review of the literature. Medicine (Baltimore). 1985;64:36-48.

5. Bodle EE, Cunningham JA, Della-Latta $P$, et al. Epidemiology of nontuberculous mycobacteria in patients without HIV infection, New York City. Emerg Infect Dis. 2008;14:290-296.

6. Boyd AS, Robbins J. Cutaneous Mycobacterium avium intracellulare infection in an HIV+ patient mimicking histoid leprosy. Am J Dermatopathol. 2005;27:39-41.

7. Griffith DE, Aksamit T, Brown-Elliott BA, et al. An official ATS/IDSA statement: diagnosis, treatment, and prevention of nontuberculous mycobacterial diseases. Am J Respir Crit Care Med. 2007;175:367-416. 